\title{
LA NEURODIDÁCTICA Y LA EVALUACIÓN FORMATIVA. DISCURSO Y RELATO DE UNA INVESTIGACIÓN
}

The neurodydactic and the formative evaluation. Speech and story of an investigation

A neurodidática e a avaliação formativa. Discurso e relato de uma investigação

\author{
Dra. María Amparo Calatayud Salom (1)
}

María Amparo Torres Bellvis (2)

(1) Profesora Titular de Universidad. Departamento de Didáctica y Organización Escolar. Facultad de Filosofía y Ciencias de la Educación. Universitat de Valencia. Valencia. España. Teléfono 963864427. E-mail: amparo.calatayud@uv.es

(2) Doctoranda del Programa de Educación de la Universitat de València. Valencia. España. Teléfono 607163613 E-mail: matobell@alumni.uv.es

\section{Resumen}

En esta comunicación se presentan no sólo los supuestos teóricos y las implicaciones metodológicas para hacer prácticas neuroevaluativas formativas sino, también, se presentan los resultados cualitativos obtenidos por una muestra de 240 estudiantes universitarios acerca de cómo han sido evaluados. Desde la mirada de los estudiantes podemos constatar que la evaluación vivida dista bastante de los supuestos impregnados por la neurociencia, así como también, por los supuestos formativos. Constatar esta realidad es necesario para darse cuenta de que ésta ha de cambiar si queremos realizar prácticas evaluativas de excelencia que estimulen la generación de neurotransmisores que garanticen adecuadas sinapsis en el cerebro de los estudiantes. Impregnar el proceso evaluativo de fundamentos neuroeducativos enriquece las prácticas de evaluación formativa.

Palabras clave: Neurociencia; evaluación; neurodidáctica; técnicas evaluativas

\begin{abstract}
This paper presents not only the theoretical assumptions and the methodological implications to make formative neuroevaluative practices a reality, but also the qualitative results obtained by a sample of 240 university students about how they have
\end{abstract}


been evaluated. From the students' point of view, we can verify that the evaluation lived is far from the assumptions impregnated by neuroscience, as well as by the formative assumptions. To verify this reality is necessary to realize that it has to change if we want to carry out evaluative practices of excellence that stimulate the generation of neurotransmitters that guarantee adequate synapses in the students' brain. Impregnate the evaluation process of neuroeducational foundations enriches the formative evaluation practices.

Keywords: Neuroscience; evaluation; neurodidactic; evaluative techniques

\section{Resumo}

Nesta comunicação apresentam-se não só os pressupostos teóricos e as implicações metodológicas para realizar práticas neuro-avaliativas formativas, como também, se apresentam os resultados qualitativos obtidos por uma amostra de 240 estudantes universitários sobre como eles foram avaliados. Tendo em conta o ponto de vista dos estudantes, podemos verificar que o modo como veem a avaliação está bastante desfasado dos pressupostos impregnados pela neurociência, assim como dos pressupostos formativos. É necessário constatar esta realidade para percebermos que é preciso mudar se quisermos realizar práticas avaliativas de excelência, que estimulem a geração de neurotransmissores que garantam sinapses adequadas no cérebro dos alunos. Os professores têm o desafio de serem modificadores do cérebro e a prática avaliativa formativa determina, sem dúvida, a estrutura, a química e a atividade elétrica do cérebro. Impregnar o processo de avaliação de fundamentos neuro educativos enriquece as práticas de avaliação formativa.

Palavras-chave: Neurociência; avaliação; neurodidática; técnicas avaliativas

\section{Introducción}

Los avances en las Neurociencias han posibilitado el acceder con una mayor exactitud a cómo aprende el cerebro. Hoy por hoy, los progresos de las neurociencias están aportando un cambio de paradigma en la educación y, por supuesto, también en la evaluación. Están surgiendo evidencias de que una nueva evaluación es necesaria y posible. Atender e introducir los supuestos de la Neurociencia nos puede ayudar a dar un giro no sólo en la concepción de la evaluación sino también en su praxis evaluativa. 
Supone practicar una evaluación formativa que forme al estudiante, se adapte a sus peculiaridades y forme también al docente (Calatayud, 2000).

Recientes investigaciones (Kalbfleish, 2012; Wassermann \& Zambo, 2013) consideran que el objetivo de la Neurodidáctica radica en responder a la diversidad de estudiantes desde el aula, es decir, desde un sistema inclusivo, creando sinapsis, enriqueciendo el número de conexiones neurales mediante interacciones que determinen el cableado neuronal y promuevan la mayor cantidad de interconexiones del cerebro. Si esta disciplina se ocupa de estudiar la optimización del proceso de enseñanza y aprendizaje, presenta sin lugar a dudas, una incidencia transcendental en el proceso evaluador.

El objetivo general de la experiencia que se presenta fue analizar las percepciones del estudiante universitario en el curso académico 2018-19 acerca de cómo han sido evaluados en sus años de escolaridad. Se pretendía constatar si la evaluación vivida distaba o no mucho de los supuestos definidos por la neurociencia y por la evaluación formativa.

\section{Método}

El método de investigación que se utilizó fue el análisis de contenido. Dicho método distingue tres tipos de análisis sobre la evaluación vivida: sintáctico (se interesa por la morfología del texto), el semántico (busca el sentido de las palabras y el análisis de los temas y categorías propuestas) y el pragmático (pretende descubrir las circunstancias en las que la comunicación sobre la evaluación vivida tiene lugar),

En este estudio han participado 240 estudiantes de primer curso del Grado de Magisterio de la Universidad de Valencia (España). Para tal fin, se diseñó un cuestionario abierto para indagar cuestiones relativas a la percepción acerca de la evaluación vivida.

\section{Resultados}

A continuación, se describen algunas de las categorías más importantes en relación con las tres cuestiones planteadas:

a-Significado de la evaluación vivida:

Las respuestas a esta pregunta inciden mayoritariamente en una concepción de la evaluación como: 
1) Herramienta que se usa para comprobar el nivel de asimilación de los conceptos.

2) Examen que se realiza de forma objetiva sobre aspectos concretos de una asignatura.

3) Es el procedimiento mediante el cual el docente examina al estudiante para comprobar que conocimientos ha adquirido y calificarlos.

4) Un conjunto de pruebas que se le pasan al alumnado para conocer si ha adquirido los conocimientos.

5) Una nota que plasma un resultado final.

6) Método mediante el cual se averigua si un estudiante ha aprendido lo que se esperaba.

Después de estas concepciones le siguen en menor medida, la idea de la evaluación como:

1) Observar y conocer los conocimientos que tiene un estudiante.

2) La valoración de conocimientos, actitud y rendimiento de una persona.

3) La forma de ver la evolución de un estudiante y ver si está aprendiendo.

4) Proceso que permite observar en qué punto del proceso de aprendizaje está el alumno para saber si sigue el ritmo adecuado de la clase.

\section{b-Procesos mentales implicados en la evaluación vivida.}

En este caso, las respuestas se organizaron de forma exclusiva en las siguientes afirmaciones: memorización, repetición, concentración, organización, empollar, vomitar todo lo estudiado. Algunos estudiantes hablan incluso de vómito cognitivo. En un índice más bajo de incidencia se presentan los siguientes procesos mentales implicados en la evaluación: pensar, relacionar conceptos, transferir los conocimientos, reflexión, comprender, entender, razonar, elaborar mapas conceptuales y aprender.

c-Instrumentos con los que se les ha evaluado.

Según los encuestados, mayoritariamente han sido evaluados a través de exámenes (tipo test, de desarrollo y orales) controles, trabajos, etc. $\mathrm{Y}$ en menor medida, a través de tareas de clase, participación, revisión del cuaderno, observación, debate en el aula, entrevistas, deberes, trabajo diario, libreta, comportamiento y actitud, asistencia, reuniones consensuadas para evidenciar el aprendizaje realizado y portafolio. 


\section{Conclusiones}

Un análisis de la evaluación vivida por los estudiantes encuestados nos ha demostrado que la evaluación está anclada en el pasado y dirigida casi, exclusivamente, a medir resultados finales de aprendizaje. Se trata de una concepción de la evaluación vinculada a una racionalidad tecnoburocrática del curriculum, en la que se priman los conocimientos, los efectos observables, la vertiente negativa, se penaliza el error, etc. Por ello, es necesario una formación del profesorado que apueste por una forma de evaluar que utilice diversas técnicas de evaluación participativas tanto para el alumnado como para el docente. Técnicas que ayuden a los estudiantes a conocer lo que saben y hasta dónde pueden llegar potenciando sus capacidades y aprovechando al máximo sus posibilidades. Por tanto, impregnar el proceso evaluativo de fundamentos neuroeducativos supone una exigencia y una necesidad hoy para las prácticas de evaluación formativas. Además, este estudio ha resultado ser muy enriquecedor para el alumnado participante en la investigación porque les ha hecho conscientes de las formas de evaluación que han experimentado, y de cómo esto puede ser útil para transformar sus ideas sobre la evaluación, avanzando hacia modelos más alineados con las neurociencias y la evaluación formativa.

\section{Referencias}

Calatayud Salom, M.A (2000)."Reflexión de los alumnos de Educación Primaria sobre preconcepciones evaluativas". AA.VV Evaluación como ayuda al aprendizaje. Barcelona: Graó-Laboratorio Educativo.

Kalbfleisch, M.L. (2012). Neurotechnology in education. New York: Eds Taylor and Francis.

Wassermann,L, \& Zambo, D. (2013). Early childhood and Neuroscience. New York: Springer. 